Revista de MATEMÁticA: TeORÍA y APliCACIONEs 2017 24(2) : 239-255

CIMPA - UCR ISSN: 1409-2433 (PRINT), 2215-3373 (ONLINE)

\title{
ON THE POST-LINEAR QUADRUPOLE-QUADRUPOLE METRIC
}

\author{
SOBRE LA MÉTRICA POST-LINEAL \\ CUADRUPOLO-CUADRUPOLO
}

FRANCisco Frutos-Alfaro* Michael SOFFEL ${ }^{\dagger}$

Received: 25/May/2016; Revised: 17/Apr/2017;

Accepted: 28/Apr/2017

Revista de Matemática: Teoría y Aplicaciones is licensed under a Creative Commons Reconocimiento-NoComercial-Compartirigual 4.0 International License.

Creado a partir de la obra en http://www.revistas.ucr.ac.cr/index.php/matematica

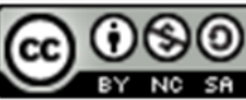

* School of Physics and Space Research Center, University of Costa Rica, San José, Costa Rica. E-Mail: frutos@ fisica.ucr.ac.cr

${ }^{\dagger}$ Technical University Dresden and Lohrmann Observatory, Dresden, Germany. E-Mail: Michael.Soffel@mailbox.tu-dresden.de 


\begin{abstract}
The Hartle-Thorne metric defines a reliable spacetime for most astrophysical purposes, for instance simulations of slowly rotating stars. Solving the Einstein field equations, we added terms of second order in the quadrupole moment to its post-linear version in order to compare it with solutions found by Blanchet in the multi-polar post-Minkowskian framework. We first derived the extended Hartle-Thorne metric in harmonic coordinates and then showed agreement with the corresponding post-linear metric from Blanchet.

We also found a coordinate transformation from the post-linear ErezRosen metric to our extended Hartle-Thorne spacetime. It is well known that the Hartle-Thorne solution can be smoothly matched with an interior perfect fluid solution with appropriate physical properties. A comparison among these solutions provides a validation of them. It is clear that in order to represent realistic solutions of self-gravitating (axially symmetric) matter distributions of perfect fluid, the quadrupole moment has to be included as a physical parameter.
\end{abstract}

Keywords: general relativity; solutions of Einstein's equations; approximation procedures; weak fields.

\title{
Resumen
}

La métrica de Hartle-Thorne define un espacio-tiempo confiable para la mayoría de propósitos astrofísicos, por ejemplo simulaciones de estrellas girando lentamente. Añadimos términos de segundo orden en el momento cuadripolar a su versión post-lineal al resolver las ecuaciones de campo de Einstein. La solución encontrada es comparada con la encontrada por Blanchet en el marco post-Minkowskiano. Primero derivamos la métrica de Hartle-Thorne en coordenadas armónicas y luego mostramos la concordancia con la correspondiente métrica post-lineal de Blanchet.

También encontramos una transformación de coordenadas de la métrica de Erez-Rosen post-lineal al espacio-tiempo de Hartle-Thorne obtenido. Es bien sabido que la solución de Hartle-Thorne puede estar suavemente acoplada con una solución de fluido interior perfecto con propiedades físicas apropiadas. Una comparación entre estas soluciones proporciona una validación de las mismas. Está claro que para representar soluciones realistas de distribuciones de materia auto-gravitantes (axialmente simétricas) de fluido perfecto, el momento cuadripolar tiene que ser incluido como un parámetro físico.

Palabras clave: relatividad general; soluciones de las ecuaciones de Einstein; procedimientos de aproximación; campos débiles.

Mathematics Subject Classification: 83C05, 83C25, 85-02. 


\section{Introduction}

In 1968, Hartle and Thorne (HT) $[16,26]$ proposed an approximate solution to the Einstein field equations (EFE) intended to represent the gravity field of neutron stars with mass, rotation and quadrupole moment as parameters. Berti et al. [1] compared the HT metric with the Manko [20, 21] (exact solutions) and Cook-Shapiro-Teukolsky metrics [6] (numerical solution), and showed that it is safe to use the HT metric, since it gives excellent results even for the innermost stable circular orbits with fast spin periods. Moreover, the exterior HT metric can be smoothly matched with a physically reasonable interior one. This provides realistic models of stars and for this reason, it is often used to validate exact and approximate solutions of EFE.

Stationary exact solutions of EFE in the vacuum case are characterized by two families of multipole-moments: mass- and spin-moments, for example see $[18,20,22,25]$. Some of these solutions might be appropriate to represent stellar objects where the field moments can be related with corresponding body moments as integrals over the field generating sources. Moreover, the postlinear approximation of these metrics must be compatible with the HT solution. Quevedo et al. [26] and Frutos et al. [11, 12] compared the HT solution with exact and approximate solutions of the EFE with a quadrupole moment $Q$ at first order. Comparisons with the second order in $Q$ of these solutions are still missing.

Geroch and Hansen (GH) defined a procedure to find the field multipole moments of static and stationary spacetimes [13, 15]. Alternative definitions of relativistic multipole moments were given by Simon and Beig [28] and by Thorne [29]. It is important to mention that the GH multipole moments are equivalent to the Thorne moments for stationary systems [14]. Using the Ernst formalism [5, 9], Fodor et al. [10] found an elegant method to find the multipole moments of a given spacetime. This method was later generalized by Hoenselaers and Perjés [19]. The relevance of taking the correct relativistic multipole moments in numerical spacetimes for modelling astrophysical objects, such as neutron stars, was discussed by Pappas and Apostolatos [23], who used a method due to Ryan [27] to derive the multipole moments.

Nowadays, the use of harmonic coordinates is customary, since the form of the transfomed metric tensor using harmonic coordinates has a special structure that allows to read off the Thorne moments even for the non-stationary case $[18,29]$. The multi-polar post-Minkowskian formalism (MPM) that was developed by Blanchet, Damour and Iyer $[2,7]$ is also formulated in harmonic coordinates. Applying this formalism, Blanchet found spacetimes containing massquadrupole and quadrupole-quadrupole terms $[3,4]$. The main goal of this paper 
is to compare their results with ours, which we got from the HT approximation with squared quadrupole moment.

The paper is organized as follows. In the second section, we briefly describe the HT metric. We find a new expanded version of the HT metric with a squared quadrupole moment in the third section. In the fourth section, the harmonic coordinates for this HT solution are obtained and the metric is expressed in these coordinates. In the fifth section, it is shown that our HT harmonic metric and the Blanchet metric coincide at our level of approximation. Finally, a coordinate transformation is found from the post-linear version of the Erez-Rosen metric $[5,8,30,31,32]$ to this HT solution with no rotation in the sixth section.

\section{The Hartle-Thorne metric}

The Hartle-Thorne metric $[16,26]$ is an approximate solution of vacuum EFE that describes the exterior of any slowly and rigidly rotating, stationary and axially symmetric body. The metric is given with accuracy up to the second order terms in the body's angular momentum, and first order in its quadrupole moment. It therefore has three parameters: mass $M$, spin $S$ and quadrupole-moment $Q$. The HT solution is given by

$$
d s^{2}=g_{t t} d t^{2}+g_{r r} d r^{2}+g_{\theta \theta} d \theta^{2}+g_{\theta \theta} \sin ^{2} \theta d \phi^{2}+2 g_{t \phi} d t d \phi,
$$

with metric components

$$
\begin{aligned}
g_{t t} & =-(1-2 U)\left[1+2 K_{1} P_{2}(\cos \theta)\right]-2 \frac{J^{2}}{r^{4}}\left(2 \cos ^{2} \theta-1\right) \\
g_{t \phi} & =-2 \frac{J}{r} \sin ^{2} \theta \\
g_{r r} & =\frac{1}{1-2 U}\left[1-2 K_{2} P_{2}(\cos \theta)-\frac{2}{1-2 U} \frac{J^{2}}{r^{4}}\right] \\
g_{\theta \theta} & =r^{2}\left[1-2 K_{3} P_{2}(\cos \theta)\right] \\
g_{\phi \phi} & =g_{\theta \theta} \sin ^{2} \theta
\end{aligned}
$$

where

$$
\begin{aligned}
& K_{1}=\frac{J^{2}}{m r^{3}}(1+U)+\frac{5}{8}\left(\frac{q}{m^{3}}-\frac{J^{2}}{m^{4}}\right) Q_{2}^{2}\left(\frac{r}{m}-1\right), \\
& K_{2}=K_{1}-\frac{6 J^{2}}{r^{4}} \\
& K_{3}=\left(K_{1}+\frac{J^{2}}{r^{4}}\right)+\frac{5}{4}\left(\frac{q}{m^{3}}-\frac{J^{2}}{m^{4}}\right) \frac{U}{\sqrt{1-2 U}} Q_{2}^{1}\left(\frac{r}{m}-1\right),
\end{aligned}
$$




$$
\begin{gathered}
m=\frac{G M}{c^{2}}, \quad J=\frac{G S}{c^{3}}, \quad q=\frac{G Q}{c^{2}}, \\
U=\frac{m}{r} \quad \text { and } \quad P_{2}(\cos \theta)=\frac{1}{2}\left[3 \cos ^{2} \theta-1\right] .
\end{gathered}
$$
kind,

The functions $Q_{2}^{1,2}$ are the associated Legendre polynomials of the second

$$
\begin{aligned}
& Q_{2}^{1}(x)=\sqrt{x^{2}-1}\left(\frac{3}{2} x \ln \left(\frac{1+x}{1-x}\right)-\frac{\left(3 x^{2}-2\right)}{\left(x^{2}-1\right)}\right), \\
& Q_{2}^{2}(x)=\left(x^{2}-1\right)\left(\frac{3}{2} \ln \left(\frac{1+x}{1-x}\right)-\frac{\left(3 x^{3}-5 x\right)}{\left(x^{2}-1\right)^{2}}\right) .
\end{aligned}
$$

\section{The post-linear Hartle-Thorne with $q^{2}$ term}

Neglecting $m^{3}, J^{2}$-terms and changing $q \rightarrow-q$ in the HT-metric one obtains

$$
\begin{aligned}
g_{t t} & =-\left(1-2 \frac{m}{r}-2 \frac{q}{r^{3}} P_{2}-2 \frac{m q}{r^{4}} P_{2}\right), \\
g_{t \phi} & =-2 \frac{J}{r} \sin ^{2} \theta \\
g_{r r} & =1+2 \frac{m}{r}+2 \frac{q}{r^{3}} P_{2}+4 \frac{m^{2}}{r^{2}}+10 \frac{m q}{r^{4}} P_{2}, \\
g_{\theta \theta} & =r^{2}\left(1+2 \frac{q}{r^{3}} P_{2}+5 \frac{m q}{r^{4}} P_{2}\right), \\
g_{\phi \phi} & =r^{2} \sin ^{2} \theta\left(1+2 \frac{q}{r^{3}} P_{2}+5 \frac{m q}{r^{4}} P_{2}\right) .
\end{aligned}
$$

We then add $q^{2}$-terms and check that the corresponding metric

$$
\begin{aligned}
g_{t t} & =-\left(1-2 \frac{m}{r}-2 \frac{q}{r^{3}} P_{2}-2 \frac{m q}{r^{4}} P_{2}+2 \frac{q^{2}}{r^{6}} P_{2}^{2}\right) \\
g_{t \phi} & =-2 \frac{J}{r} \sin ^{2} \theta \\
g_{r r} & =1+2 \frac{m}{r}+2 \frac{q}{r^{3}} P_{2}+4 \frac{m^{2}}{r^{2}}+10 \frac{m q}{r^{4}} P_{2}-\frac{1}{12} \frac{q^{2}}{r^{6}}\left[-8 P_{2}^{2}+16 P_{2}-77\right], \\
g_{\theta \theta} & =r^{2}\left(1+2 \frac{q}{r^{3}} P_{2}+5 \frac{m q}{r^{4}} P_{2}-\frac{1}{36} \frac{q^{2}}{r^{6}}\left[-44 P_{2}^{2}-8 P_{2}+43\right]\right) \\
g_{\phi \phi} & =r^{2} \sin ^{2} \theta\left(1+2 \frac{q}{r^{3}} P_{2}+5 \frac{m q}{r^{4}} P_{2}-\frac{1}{36} \frac{q^{2}}{r^{6}}\left[-44 P_{2}^{2}-8 P_{2}+43\right]\right)
\end{aligned}
$$

is solution of the EFE by means of a REDUCE program [17]. 


\section{Transformation to harmonic coordinates}

Harmonic coordinates $(c T, X, Y, Z)$ are especially useful, because the form of the metric tensor in these coordinates has a special structure where the Thornemoments can be read off even for the non-stationary case [18, 29]. The harmonic coordinate condition reads

$$
\square X^{\mu}=g^{\alpha \beta} \frac{\partial^{2} X^{\mu}}{\partial x^{\alpha} \partial x^{\beta}}-g^{\alpha \beta} \Gamma_{\alpha \beta}^{\nu} \frac{\partial X^{\mu}}{\partial x^{\nu}}=0 .
$$

The solution of this equation for the HT metric including $q^{2}$-terms reads

$$
\begin{aligned}
T & =t, \\
X & =f \sin \theta \cos \phi=R \sin \vartheta \cos \phi, \\
Y & =f \sin \theta \sin \phi=R \sin \vartheta \sin \phi, \\
Z & =h \cos \theta=R \cos \vartheta,
\end{aligned}
$$

where

$$
\begin{aligned}
f=[r-m+ & \left.\frac{1}{2} \frac{m q}{r^{3}} \cos ^{2} \theta+\frac{1}{72} \frac{q^{2}}{r^{5}}\left(32 P_{2}^{2}-4 P_{2}-55\right)\right], \\
h=[r-m- & \left.\frac{1}{2} \frac{m q}{r^{3}} \sin ^{2} \theta+\frac{1}{72} \frac{q^{2}}{r^{5}}\left(32 P_{2}^{2}-16 P_{2}-43\right)\right], \\
R^{2} & =X^{2}+Y^{2}+Z^{2} \\
& \simeq[r-m]^{2}+\frac{1}{12} \frac{q^{2}}{r^{4}}\left[8 P_{2}^{2}-17\right], \\
r & \simeq R+m-\frac{1}{24} \frac{q^{2}}{R^{5}}\left[8 P_{2}^{2}-17\right], \\
\tan \vartheta & =\frac{\sqrt{X^{2}+Y^{2}}}{Z}=\frac{f}{h} \tan ^{2} \\
& \simeq\left[1+\frac{1}{2} \frac{m q}{r^{4}}-\frac{1}{4} \frac{q^{2}}{r^{6}} \sin ^{2} \theta\right] \tan \theta \\
\theta & \simeq \vartheta-\frac{1}{2}\left[\frac{m q}{R^{4}}-\frac{1}{2} \frac{q^{2}}{R^{6}} \sin ^{2} \vartheta\right] .
\end{aligned}
$$

The transformation of the metric from coordinates $(r, \theta, \phi)$ to such harmonic coordinates $(R, \vartheta, \phi)$, was performed by means of the differential 1forms $(d X, d Y, d Z)$. From these 1-forms, it is solved for the other 1-forms $(d r, d \theta, d \phi)$ : 


$$
\begin{aligned}
d r & =\left(1-\frac{1}{r}\left(\alpha_{1}-\alpha_{2}\right) \cos ^{2} \theta-\frac{\partial \alpha_{1}}{\partial r} \sin ^{2} \theta-\frac{\partial \alpha_{2}}{\partial r} \cos ^{2} \theta\right) \\
& \times(\sin \theta(\cos \phi d X+\sin \phi d Y)+\cos \theta d Z) \\
& +\frac{1}{r}\left(\frac{\partial \alpha_{1}}{\partial \theta} \sin ^{2} \theta+\frac{\partial \alpha_{1}}{\partial \theta} \cos ^{2} \theta\right)(\sin \theta d Z-\cos \theta(\cos \phi d X+\sin \phi d Y)) \\
& +\frac{1}{r}\left(\alpha_{1}-\alpha_{2}\right) \cos \theta d Z \\
r d \theta & =\left(1+U+U^{2}-\frac{1}{r}\left(\alpha_{1} \cos ^{2} \theta+\alpha_{2} \sin ^{2} \theta\right)-\left(\frac{\partial \alpha_{1}}{\partial r}-\frac{\partial \alpha_{1}}{\partial r}\right) \sin ^{2} \theta\right) \\
& \times(\cos \theta(\cos \phi d X+\sin \phi d Y)-\sin \theta d Z) \\
& -\frac{1}{r} \sin \theta\left(\frac{\partial \alpha_{1}}{\partial \theta}-\frac{\partial \alpha_{2}}{\partial \theta}\right)\left(\cos ^{2} \theta(\cos \phi d X+\sin \phi d Y)-\cos \theta \sin \theta d z\right) \\
& -\left(\frac{\partial \alpha_{1}}{\partial r}-\frac{\partial \alpha_{1}}{\partial r}\right) \sin \theta d Z
\end{aligned}
$$

$r \sin \theta d \phi=\left(1+U+U^{2}-\frac{\alpha_{1}}{r}\right)(\cos \phi d Y-\sin \phi d X)$

where

$$
\begin{aligned}
\alpha_{1} & =\frac{1}{2} \frac{m q}{r^{3}} \cos ^{2} \theta+\frac{1}{72} \frac{q^{2}}{r^{5}}\left(32 P_{2}^{2}-4 P_{2}-55\right) \\
\alpha_{2} & =-\frac{1}{2} \frac{m q}{r^{3}} \sin ^{2} \theta+\frac{1}{72} \frac{q^{2}}{r^{5}}\left(32 P_{2}^{2}-16 P_{2}-43\right) .
\end{aligned}
$$

Substituting (8), (9), and (10) into the metric (4), the metric in Cartesian coordinates to post-linear order takes the form

$$
d s^{2}=g_{t t} d t^{2}+2 g_{t i} d t d X^{i}+g_{i j} d X^{i} d X^{j}
$$


where

$$
\begin{aligned}
& g_{t t}=-1+2 \frac{w}{c^{2}}-2 \frac{w^{2}}{c^{4}} \\
& g_{t X}=2 \frac{J}{R^{3}} Y \\
& g_{t Y}=-2 \frac{J}{R^{3}} X \\
& g_{X X}=1+2 \frac{w}{c^{2}}+2 \frac{w^{2}}{c^{4}}+\left(\frac{X^{2}}{R^{2}}-1\right) \frac{m^{2}}{R^{2}} \\
& +\frac{1}{2} \frac{m q}{R^{4}}\left(1-\frac{X^{2}}{R^{2}}-5 \frac{Z^{2}}{R^{2}}+15 \frac{X^{2} Z^{2}}{R^{4}}\right) \\
& +\frac{q^{2}}{4 R^{6}}\left(-1+3 \frac{X^{2}}{R^{2}}+12 \frac{Z^{2}}{R^{2}}-54 \frac{X^{2} Z^{2}}{R^{4}}-15 \frac{Z^{4}}{R^{4}}+75 \frac{X^{2} Z^{4}}{R^{6}}\right), \\
& g_{X Y}=m^{2} \frac{X Y}{R^{4}}+\frac{1}{2} m q \frac{X Y}{R^{6}}\left(-1+15 \frac{Z^{2}}{R^{2}}\right)+\frac{3}{4} q^{2} \frac{X Y}{R^{8}}\left(1-18 \frac{Z^{2}}{R^{2}}+25 \frac{Z^{4}}{R^{4}}\right) \text {, } \\
& g_{X Z}=m^{2} \frac{X Z}{R^{4}}+\frac{1}{2} m q \frac{X Z}{R^{6}}\left(-7+15 \frac{Z^{2}}{R^{2}}\right)+\frac{3}{4} q^{2} \frac{X Z}{R^{8}}\left(5-26 \frac{Z^{2}}{R^{2}}+25 \frac{Z^{4}}{R^{4}}\right) \text {, } \\
& g_{Y Y}=1+2 \frac{w}{c^{2}}+2 \frac{w^{2}}{c^{4}}+\left(\frac{Y^{2}}{R^{2}}-1\right) \frac{m^{2}}{R^{2}} \\
& +\frac{1}{2} \frac{m q}{R^{4}}\left(1-\frac{Y^{2}}{R^{2}}-5 \frac{Z^{2}}{R^{2}}+15 \frac{Y^{2} Z^{2}}{R^{4}}\right) \\
& +\frac{q^{2}}{4 R^{6}}\left(-1+3 \frac{Y^{2}}{R^{2}}+12 \frac{Z^{2}}{R^{2}}-54 \frac{Y^{2} Z^{2}}{R^{4}}-15 \frac{Z^{4}}{R^{4}}+75 \frac{Y^{2} Z^{4}}{R^{6}}\right), \\
& g_{Y Z}=m^{2} \frac{Y Z}{R^{4}}+\frac{1}{2} m q \frac{Y Z}{R^{6}}\left(-7+15 \frac{Z^{2}}{R^{2}}\right)+\frac{3}{4} q^{2} \frac{Y Z}{R^{8}}\left(5-26 \frac{Z^{2}}{R^{2}}+25 \frac{Z^{4}}{R^{4}}\right) \text {, } \\
& g_{Z Z}=1+2 \frac{w}{c^{2}}+2 \frac{w^{2}}{c^{4}}+\left(\frac{Z^{2}}{R^{2}}-1\right) \frac{m^{2}}{R^{2}} \\
& +\frac{3}{2} \frac{m q}{R^{4}}\left(1-6 \frac{Z^{2}}{R^{2}}+5 \frac{Z^{4}}{R^{4}}\right) \\
& +\frac{3}{4} \frac{q^{2}}{R^{6}}\left(-1+15 \frac{Z^{2}}{R^{2}}-39 \frac{Z^{4}}{R^{4}}+25 \frac{Z^{6}}{R^{6}}\right),
\end{aligned}
$$




$$
\begin{aligned}
g_{Y Z} & =m^{2} \frac{Y Z}{R^{4}}+\frac{1}{2} m q \frac{Y Z}{R^{6}}\left(-7+15 \frac{Z^{2}}{R^{2}}\right)+\frac{3}{4} q^{2} \frac{Y Z}{R^{8}}\left(5-26 \frac{Z^{2}}{R^{2}}+25 \frac{Z^{4}}{R^{4}}\right), \\
g_{Z Z} & =1+2 \frac{w}{c^{2}}+2 \frac{w^{2}}{c^{4}}+\left(\frac{Z^{2}}{R^{2}}-1\right) \frac{m^{2}}{R^{2}} \\
& +\frac{3}{2} \frac{m q}{R^{4}}\left(1-6 \frac{Z^{2}}{R^{2}}+5 \frac{Z^{4}}{R^{4}}\right) \\
& +\frac{3}{4} \frac{q^{2}}{R^{6}}\left(-1+15 \frac{Z^{2}}{R^{2}}-39 \frac{Z^{4}}{R^{4}}+25 \frac{Z^{6}}{R^{6}}\right),
\end{aligned}
$$

where

$$
w=\frac{G M}{R}+\frac{G Q}{R^{3}} P_{2}(\cos \vartheta),
$$

equation (7) was used, and

$$
P_{2}(\cos \vartheta)=\frac{1}{2}\left(3 \cos ^{2} \vartheta-1\right)=\frac{1}{2}\left(3 \frac{Z^{2}}{R^{2}}-1\right) .
$$

\section{Comparison with known results}

Our metric (12) can directly be compared with the one in Blanchet [3, 4] that was derived within the MPM-formalism which works with a tensor field

$$
h_{\alpha \beta}=\mathfrak{g}_{\alpha \beta}-\eta_{\alpha \beta}
$$

where

$$
\mathfrak{g}_{\alpha \beta}=\sqrt{-g} g_{\alpha \beta} \quad \text { and } \quad g=\operatorname{det} g_{\alpha \beta},
$$

and uses the Landau-Lifshitz form of the field equations in harmonic gauge (e.g. see [4] for more details).

The post-linear metric components under this harmonic coordinates can be written in the following form

$$
\begin{aligned}
g_{t t} & =-\left(1-\frac{2}{c^{2}} w+\frac{2}{c^{4}} w^{2}\right), \\
g_{t i} & =g_{i t}=-\frac{4}{c^{3}} w_{i}, \\
g_{i j} & =\left(1+\frac{2}{c^{2}} w+\frac{2}{c^{4}} w^{2}\right)-\frac{4}{c^{4}} h_{i j} .
\end{aligned}
$$


The potential $w$ is defined in (13), and

$$
w_{i}=\frac{2 G}{r^{2}} \epsilon_{i j k} S_{j} n_{k}
$$

where $S_{i}$ is the total angular momentum of the object, and $n_{i} \equiv x^{i} / r$ with $r^{2}=x^{2}+y^{2}+z^{2}$. In our case, we only have the $z$ component, i. e. $S_{z}=$ $S\left(J=G S / c^{3}\right)$.

The mass-quadrupole and quadrupole-quadrupole metric components as obtained by Blanchet $[3,4]$ take the form

$$
\begin{aligned}
h_{00}^{M M_{a b}} & =-\frac{21 M}{r^{4}} \hat{n}_{a b} M_{a b}, \\
h_{i j}^{M M_{a b}} & =\frac{M}{r^{4}}\left(-\frac{15}{2} n_{i j a b} M_{a b}-\frac{1}{2} \delta_{i j} n_{a b} M_{a b}+6 n_{a(i} M_{j) a}-M_{i j}\right), \\
h_{00}^{M_{a b} M_{a b}} & =\frac{1}{r^{6}}\left(a_{0}^{6} \hat{n}_{a b c d} M_{a b} M_{c d}+b_{0}^{6} \hat{n}_{a b} M_{a c} M_{b c}+c_{0}^{6} M_{a b} M_{a b}\right), \\
h_{i j}^{M_{a b} M_{a b}} & =\frac{1}{r^{6}}\left(p_{0}^{6} \hat{n}_{i j a b c d} M_{a b} M_{c d}+q_{0}^{6} \hat{n}_{i j a b} M_{a c} M_{b c}+r_{0}^{6} \delta_{i j} \hat{n}_{a b c d} M_{a b} M_{c d}\right. \\
& +s_{0}^{6} \hat{n}_{i j} M_{a b} M_{a b}+t_{0}^{6} \delta_{i j} \hat{n}_{a b} M_{a c} M_{b c}+u_{0}^{6} \delta_{i j} M_{a b} M_{a b}+v_{0}^{6} \hat{n}_{a b c(i} M_{j) a} M_{b c} \\
& \left.+w_{0}^{6} \hat{n}_{a(i} M_{j) b} M_{a b}+x_{0}^{6} \hat{n}_{a b} M_{i j} M_{a b}+y_{0}^{6} \hat{n}_{a b} M_{a(i} M_{j) b}+z_{0}^{6} M_{a(i} M_{j) a}\right),
\end{aligned}
$$

where

$$
\begin{aligned}
& a_{0}^{6}=-\frac{63}{4}, b_{0}^{6}=-9, \quad c_{0}^{6}=-\frac{21}{10}, \\
& p_{0}^{6}=-\frac{75}{4}, \quad q_{0}^{6}=\frac{90}{11}, \quad r_{0}^{6}=-\frac{9}{44}, \\
& s_{0}^{6}=\frac{25}{84}, \quad t_{0}^{6}=-\frac{29}{42}, \quad u_{0}^{6}=-\frac{11}{70}, \\
& v_{0}^{6}=-\frac{18}{11}, \quad w_{0}^{6}=\frac{5}{21}, \quad x_{0}^{6}=-\frac{10}{21}, \\
& y_{0}^{6}=\frac{23}{42}, \quad z_{0}^{6}=-\frac{6}{35},
\end{aligned}
$$

and $\hat{n}_{i_{1} \ldots i_{l}}$ are the symmetric and trace-free parts (e.g., [24, 29]) of the Cartesian tensor

$$
n_{i_{1} \ldots i_{l}} \equiv n_{i_{1}} \cdots n_{i_{l}} .
$$

For our axially symmetric body the quadrupole-moment $Q$ appears in the Cartesian quadrupole mass-tensor in the form

$$
M_{i j}=-\frac{Q}{3}\left(\delta_{i j}-3 \delta_{i 3} \delta_{j 3}\right)
$$


Substituting these $M_{a b}$ components into (16) and the resulting $h_{\alpha \beta}$ into (15), we get the metric components (12).

\section{Transformation from Erez-Rosen to Hartle-Thorne}

The Erez-Rosen (ER) metric represents an axial symmetric static exact solution of EFE with mass and quadrupole moment [5, 8, 30, 31, 32]. It is given by

$d s^{2}=-\mathrm{e}^{2 \psi} d t^{2}+\mathrm{e}^{2(\gamma-\psi)} \Delta\left(\frac{d r^{2}}{r^{2}-2 m r}+d \theta^{2}\right)+\mathrm{e}^{-2 \psi}\left(r^{2}-2 m r\right) \sin ^{2} \theta d \phi^{2}$,

where $m$ is the mass of the object $\left(m=G M / c^{2}\right.$ ) and

$$
\Delta=r^{2}-2 m r+m^{2} \sin ^{2} \theta .
$$

The fields $\psi$ and $\gamma$ in prolate spheroidal coordinates are

$$
\begin{aligned}
\psi & =\frac{1}{2}\left\{\left(1+\frac{\tilde{q}}{4}\left(3 x^{2}-1\right)\left(3 y^{2}-1\right)\right) \ln \left[\frac{x-1}{x+1}\right]\right. \\
& \left.+\frac{3}{2} \tilde{q} x\left(3 y^{2}-1\right)\right\} \\
\gamma & =\frac{1}{2}(1+\tilde{q})^{2} \ln \left[\frac{x^{2}-1}{x^{2}-y^{2}}\right] \\
& -\frac{3}{2} \tilde{q}\left(1-y^{2}\right)\left[x \ln \left[\frac{x-1}{x+1}\right]+2\right] \\
& +\frac{9}{4} \tilde{q}^{2}\left(1-y^{2}\right)\left[\frac{1}{16}\left(x^{2}-1\right)\left(x^{2}+y^{2}-9 x^{2} y^{2}-1\right) \ln ^{2}\left[\frac{x-1}{x+1}\right]\right. \\
& +\frac{x}{4}\left(x^{2}+7 y^{2}-9 x^{2} y^{2}-\frac{5}{3}\right) \ln \left[\frac{x-1}{x+1}\right] \\
& \left.+\frac{x^{2}}{4}\left(1-9 y^{2}\right)+\left(y^{2}-\frac{1}{3}\right)\right] .
\end{aligned}
$$

with $x=r / m-1$ and $y=\cos \theta$. The quadrupole moment $q$ is given in terms of the quadrupole parameter $\tilde{q}=15 q / 2 m^{3}\left(q=G Q / c^{2}\right)$.

Now, substituting this $\tilde{q}$ and expanding in Taylor series keeping only $m^{2}$ and 
$q^{2}$ terms, the ER metric in spherical coordinates $(c t, r, \theta, \phi)$ reads

$$
\begin{aligned}
g_{t t} & =-\left(1-2 U-2 U_{q} P_{2}-2 U U_{q} P_{2}+2 U_{q}^{2} P_{2}^{2}\right), \\
g_{r r} & =1+2 U+4 U^{2}+2 U_{q} P_{2}+\frac{2}{3} U U_{q}\left(5 P_{2}^{2}+11 P_{2}-1\right) \\
& +\frac{2}{9} U_{q}^{2}\left(25 P_{2}^{3}-12 P_{2}^{2}-6 P_{2}+2\right), \\
g_{\theta \theta} & =r^{2}\left(1+2 U_{q} P_{2}+\frac{2}{3} U U_{q}\left(5 P_{2}^{2}+5 P_{2}-1\right)\right. \\
& \left.+\frac{2}{9} U_{q}^{2}\left(25 P_{2}^{3}-12 P_{2}^{2}-6 P_{2}+2\right)\right), \\
g_{\phi \phi} & =r^{2} \sin ^{2} \theta\left(1+2 U_{q} P_{2}+6 U U_{q} P_{2}+2 U_{q}^{2} P_{2}^{2}\right),
\end{aligned}
$$

where

$$
U=\frac{m}{r} \quad \text { and } \quad U_{q}=\frac{q}{r^{3}} .
$$

The following transformation converts the truncated ER metric into the static HT metric at the same level of approximation

$$
\begin{aligned}
r & =R\left(1+\frac{m q}{R^{4}} f_{1}+\frac{q^{2}}{R^{6}} f_{2}\right) \\
\theta & =\Theta+\frac{m q}{R^{4}} g_{1}+\frac{q^{2}}{R^{6}} g_{2},
\end{aligned}
$$

where

$$
\begin{aligned}
f_{1} & =\frac{1}{18}\left(10 P_{2}^{2}-8 P_{2}-2\right), \\
f_{2} & =\frac{1}{72}\left(40 P_{2}^{3}-24 P_{2}^{2}-43\right), \\
g_{1} & =-\frac{1}{6}\left(5 P_{2}-2\right) \cos \Theta \sin \Theta, \\
g_{2} & =\frac{P_{2}}{36}\left(12-30 P_{2}\right) \cos \Theta \sin \Theta,
\end{aligned}
$$

with $P_{2}=P_{2}(\cos \Theta)$. 
The transformed metric components are given by

$$
\begin{aligned}
g_{t t} & =-\left(1-2 \mathcal{U}-2 \mathcal{U}_{q} P_{2}-2 \mathcal{U} \mathcal{U}_{q} P_{2}+2 \mathcal{U}_{q}^{2} P_{2}^{2}\right) \\
g_{R \Theta} & \simeq 0 \\
g_{R R} & =1+2 \mathcal{U}+4 \mathcal{U}^{2}+2 \mathcal{U}_{q} P_{2}+10 \mathcal{U} \mathcal{U}_{q} P_{2}+\frac{1}{12} \mathcal{U}_{q}^{2}\left(8 P_{2}^{2}-16 P_{2}+77\right),(24) \\
g_{\Theta \Theta} & =R^{2}\left(1+2 \mathcal{U}_{q} P_{2}+5 \mathcal{U} \mathcal{U}_{q} P_{2}+\frac{1}{36} \mathcal{U}_{q}^{2}\left(44 P_{2}^{2}+8 P_{2}-43\right)\right), \\
g_{\phi \phi} & =R^{2} \sin ^{2} \Theta\left(1+2 \mathcal{U}_{q} P_{2}+5 \mathcal{U} \mathcal{U}_{q} P_{2}+\frac{1}{36} \mathcal{U}_{q}^{2}\left(44 P_{2}^{2}+8 P_{2}-43\right)\right),
\end{aligned}
$$

where $\mathcal{U}=m / R$ and $\mathcal{U}_{q}=q / R^{3}$. The metric components (24) correspond to the HT metric components (4) in $(t, R, \Theta, \phi)$ coordinates.

\section{Conclusions}

We expanded the HT-metric and kept only linear terms in the rotation parameter and quadratic terms in the mass parameter. Then we included second order terms in the quadrupole parameter by solving perturbatively the EFE in vacuum. We then showed that this form of the metric agrees with a corresponding metric that was derived within the MPM-formalism by Blanchet at the same order of approximation.

A transformation linking our static HT solution with the ER metric expanded in Taylor series was also found. This provides a validation of all these metrics. The quadrupole moment is an important feature which is included as a physical parameter in all these solutions.

These spacetimes can be used to represent realistic solutions of self-gravitating (axially symmetric) mass distribution of perfect fluid. This is because the HT solution can be smoothly matched with interior perfect fluid solution with reasonable physical properties, such as mass, angular velocity, and quadrupole moment.

The influence of the quadrupole moment in the gravitational lens effect due to compact objects could be investigated with this HT approximation. From this spacetime, the quadrupole moment could be introduced into the gravitational lens theory in a natural manner by calculating the Einstein deflection angle.

Nowadays, some researchers argue that the HT metric is not good enough to model neutron stars, because it has no spin octupole. The HT spacetime could be improved introducing this spin octupole by perturbing it as we did with the second order of the quadrupole moment. 


\section{References}

[1] Berti, E.; White, F.; Maniopoulou, A.; Bruni, M. (2005) "Rotating neutron stars: An invariant comparison of approximate and numerical spacetime models", Monthly Notices of the Royal Astronomical Society 358: 923-938. http://dx.doi.org/10.1111/j.1365-2966.2005. $08812 . x$

[2] Blanchet, L.; Damour, T. (1986) "Radiative gravitational fields in general relativity I. General structure of the field outside the source", Philosophical Transactions of the Royal Society of London A 320(1555): 379-430. http://dx.doi.org/10.1098/rsta.1986.0125

[3] Blanchet, L. (1998) "Quadrupole-quadrupole gravitational waves", Classical and Quantum Gravity 15(1): 89-111. http://dx.doi.org/10 . $1088 / 0264-9381 / 15 / 1 / 008$

[4] Blanchet, L. (2014) "Gravitational radiation from Post-Newtonian sources and inspiralling compact binaries", Living Reviews in Relativity 17(2). http: / / www.livingreviews.org/lrr-2014-2

[5] Carmeli, M. (2001) Classical Fields: General Relativity and Gauge Theory. World Scientific Publishing, Singapore. http://www. worldscientific.com/worldscibooks/10.1142/4843

[6] Cook, G.B.; Shapiro, S.L.; Teukolsky, S A. (1994) "Rapidly rotation neutron stars in general relativity: Realistic equations of state", Astrophysical Journal 424: 823-845. http: //dx.doi.org/10.1086/173934

[7] Damour, T.; Iyer, B. (1991) "Multipole analysis for electromagnetism and linearized gravity with irreducible Cartesian tensors", Physical Review D 43(10): 3259-3272. http://dx.doi.org/10.1103/PhysRevD. 43.3259

[8] Doroshkevich, A.G.; Zel'dovich, Ya.B.; Novikov, I.D. (1966) "Gravitational collapse of nonsymmetric and rotating masses", Journal of Experimental and Theoretical Physics (Soviet Physics JETP) 22(1): 122-130. http://www.jetp.ac.ru/cgi-bin/e/index/e/22/ $1 / \mathrm{p} 122$ ? a $=1$ ist

[9] Ernst. F.J. (1968) "New formulation of the axially symmetric gravitational field problem", Physical Review 167(5): 1175-1177. http: / / dx.doi . org/10.1103/PhysRev.167.1175 
[10] Fodor, G.; Hoenselaer, C.; Perjés, Z. (1989) "Multipole moments of axisymmetric systems in relativity", Journal of Mathematical Physics 30(10): 2252-2257. http://dx.doi.org/10.1063/1.528551

[11] Frutos-Alfaro, F.; Retana-Montenegro, E.; Cordero-García, I.; BonattiGonzález, J. (2013) "Metric of a slow rotating body with quadrupole moment from the Erez-Rosen metric", International Journal of Astronomy and Astrophysics 3: 431-437. (ArXiv:1209.6126v2). http: / / dx. doi . org/10.4236/ijaa.2013.34051

[12] Frutos-Alfaro, F.; Montero-Camacho, P.; Araya, M.; Bonatti-González, J. (2015) "Approximate metric for a rotating deformed mass", International Journal of Astronomy and Astrophysics 5: 1-10. http://dx. doi.org/10.4236/ijaa.2015.51001

[13] Geroch, R. (1970) "Multipole moments. II. Curved space", Journal of Mathematical Physics 11(8): 2580-2588. http: //dx.doi.org/10. $1063 / 1.1665427$

[14] Gürsel, Y. (1983) "Multipole moments for stationary systems: The equivalence of the Geroch-Hansen formulation and the Thorne formulation", General Relativity and Gravitation 15(8): 737-754. http: / / dx. doi . org/10.1007/BF01031881

[15] Hansen, R.O. (1974) "Multipole moments of stationary space-times", Journal of Mathematical Physics 15(1): 46-52. http: / / dx . doi org/ 10 . $1063 / 1.1666501$

[16] Hartle, J.B.; Thorne K.S. (1968) "Slowly rotating relativistic stars. II. Models for neutron stars and supermassive stars", Astrophysical Journal 153: 807-834. http://dx.doi.org/10.1086/149707

[17] Hearn, A.C. (1999) REDUCE (User's and Contributed Packages Manual). Konrad-Zuse-Zentrum für Informationstechnik, Berlin. http: //www . reduce-algebra.com/docs/reduce.pdf

[18] Hernández-Pastora, J.L.; Martín, J. (1994) "Monopole-quadrupole static axisymmetric solutions of Einstein field equations", General Relativity and Gravitation 26(9): 877-907. http://dx.doi.org/10.1007/ $\mathrm{BF} 02107146$

[19] Hoenselaers, C.; Perjés, Z. (1990) "Multipole moments of axisymmetric electrovacuum spacetimes", Classical and Quantum Gravity 7(10): 18191825. http://dx.doi.org/10.1088/0264-9381/7/10/012 
[20] Manko, V.S.; Mielke, E.W.; Sanabria-Gómez, J.D. (2000) "Exact solution for the exterior field of a rotating neutron star", Physical Review D 61, 081501(R). http://dx.doi.org/10.1103/PhysRevD.61. 081501

[21] Manko, V.S.; Sanabria-Gómez, J.D.; Manko, O.V. (2000) "Nineparameter electrovac metric involving rational functions", Physical Review D 62, 044048. http: / /dx.doi.org/10.1103/PhysRevD. 62.044048

[22] Pachón, L.A.; Rueda, J.A.; Sanabria-Gómez, J.D. (2006) "Realistic exact solution for the exterior field of a rotating neutron star", Physical Review D 73, 104038. http://dx.doi.org/10.1103/PhysRevD. 73.104038

[23] Pappas, G.; Apostolatos, T.A. (2012) "Revising the multipole moments of numerical spacetimes and its consequences", Physical Review Letters 108(23), id. 231104. http://dx.doi.org/10.1103/ PhysRevLett.108.231104

[24] Poisson, E.; Will, C.M. (2014) Gravity (Newtonian, Post-Newtonian, Relativistic). Cambridge University Press, Cambridge. http://www. cambridge.org/us/academic/subjects/physics/

[25] Quevedo, H.; Mashhoon, B. (1991) "Generalization of Kerr spacetime", Physical Review 43(12): 3902-3906. http: / / dx.doi.org/ $10.1103 /$ PhysRevD . 43.3902

[26] Quevedo, H. (2011) "Exterior and interior metrics with quadrupole moment", General Relativity and Gravitation, 43(4): 1141-1152. http: / / dx.doi.org/10.1007/s10714-010-0940-5

[27] Ryan, F.D. (1995) "Gravitational waves from the inspiral of a compact object into a massive, axisymmetric body with arbitrary multipole moments", Physical Review D 52, 5707. http://dx.doi.org/10. 1103 /PhysRevD . 52.5707

[28] Simon, W.; Beig, R. (1983) "The multipole structure of stationary spacetimes", Journal of Mathematical Physics 24(5): 1163-1171. http:// dx.doi.org/10.1063/1.525846

[29] Thorne, K.S. (1980) "Multipole expansions of gravitational radiation", Reviews on Modern Physics 52(2): 299-340. http: / / dx. doi . org/10. $1103 /$ RevModPhys.52.299 
[30] Winicour, J.; Janis, A.I.; Newman, E.T. (1968) "Static, axially symmetric point horizons", Physical Review 176: 1507-1513. http://dx.doi . org/10.1103/PhysRev.176.1507

[31] Young, J.H.; Coulter; C.A. (1969) "Exact metric for a nonrotating mass with a quadrupole moment", Physical Review 184: 1313-1315. http: // dx.doi.org/10.1103/PhysRev.184.1313

[32] Zel'dovich, Ya.B.; Novikov, I.D. (2011) Stars and Relativity. Dover Publications, New York. http://store.doverpublications.com/ $0486694240 . \mathrm{html}$ 
